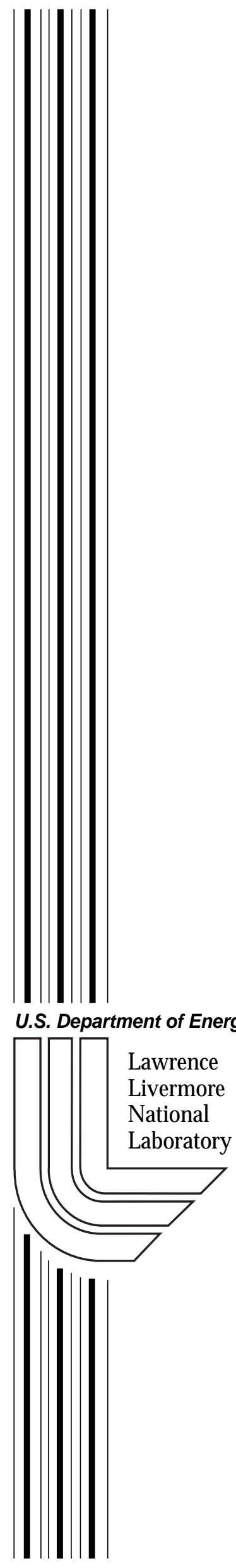

Preprint

UCRL-JC-151599

\title{
Bulk Materials Analysis using High-Energy Positron Beams
}

S.C. Glade, P. Asoka-Kumar, T.G. Nieh, P.A. Sterne, B.D. Wirth, R.H. Dauskardt, K.M. Flores, D. Suh, G.R. Odette

This article was submitted to $17^{\text {th }}$ International Conference on the Applications of Accelerators in Research and Industry, CAARI 2002, Denton, Texas, November 1216,2002

November 11, 2002 


\section{DISCLAIMER}

This document was prepared as an account of work sponsored by an agency of the United States Government. Neither the United States Government nor the University of California nor any of their employees, makes any warranty, express or implied, or assumes any legal liability or responsibility for the accuracy, completeness, or usefulness of any information, apparatus, product, or process disclosed, or represents that its use would not infringe privately owned rights. Reference herein to any specific commercial product, process, or service by trade name, trademark, manufacturer, or otherwise, does not necessarily constitute or imply its endorsement, recommendation, or favoring by the United States Government or the University of California. The views and opinions of authors expressed herein do not necessarily state or reflect those of the United States Government or the University of California, and shall not be used for advertising or product endorsement purposes.

This is a preprint of a paper intended for publication in a journal or proceedings. Since changes may be made before publication, this preprint is made available with the understanding that it will not be cited or reproduced without the permission of the author.

This report has been reproduced directly from the best available copy.

Available electronically at http://www.doe.gov/bridge

Available for a processing fee to U.S. Department of Energy

and its contractors in paper from

U.S. Department of Energy

Office of Scientific and Technical Information

P.O. Box 62

Oak Ridge, TN 37831-0062

Telephone: (865) 576-8401

Facsimile: (865) 576-5728

E-mail: reports@adonis.osti.gov

Available for the sale to the public from

U.S. Department of Commerce

National Technical Information Service

5285 Port Royal Road

Springfield, VA 22161

Telephone: (800) 553-6847

Facsimile: (703) 605-6900

E-mail: orders@ntis.fedworld.gov

Online ordering: http://www.ntis.gov/ordering.htm

OR

Lawrence Livermore National Laboratory

Technical Information Department's Digital Library

http://www.llnl.gov/tid/Library.html 


\title{
Bulk materials analysis using high-energy positron beams
}

\author{
S.C. Glade', P. Asoka-Kumar'1, T.G. Nieh'1, P.A. Sterne', B.D. Wirth'1, R.H. Dauskardt'2, K.M. Flores ${ }^{2}$, D. \\ Suh'2, and G.R. Odette
}

1Lawrence Livermore $N$ ational Laboratory, Mail Stop L-280, Livermore, CA 94550, USA, 2Stanford University, Department of $M$ aterials Science and Engineering, Stanford, CA 94305, U SA, 3U niversity of California Santa Barbara, Department of M echanical and Environmental Engineering, Santa Barbara, CA 93106, USA.

\begin{abstract}
This article reviews some recent materials analysis results using high-energy positron beams at Lawrence Livermore National Laboratory. We are combining positron lifetime and orbital electron momentum spectroscopic methods to provide electron number densities and electron momentum distributions around positron annihilation sites. Topics covered include: correlation of positron annihilation characteristics with structural and mechanical properties of bulk metallic glasses, compositional studies of embrittling features in nuclear reactor pressure vessel steel, pore characterization in Zeolites, and positron annihilation characteristics in alkali halides.
\end{abstract}

\section{Introduction}

Positrons offer a unique characterization probe for materials science. A positron is the antiparticle of an electron, having the same mass $\left(511 \mathrm{keV} / \mathrm{c}^{2}\right)$ as an electron, but a positive charge. A positron will annihilate with an electron, producing two $511 \mathrm{keV}$ gamma rays traveling in opposite directions. By measuring the timing of the gamma ray emission or the energies of these two gamma rays after annihilation of a positron in a material, one can obtain information regarding the defects and open volume regions of a material [1] as well as the elements associated with these features.

Theoretical calculations of positron behavior in materials must be combined with the experimental data to realize the full utility of positron annihilation spectroscopy (PAS). Theoretical calculations of positron lifetimes for metals and semiconductors correspond well with experimentally measured lifetimes. Theoretical predictions of lifetime values are not as reliable for wide bad gap insulators due to the lack of a quantitative understanding of strong-electron positron correlation effects in these systems. A review of positron theory can be found in Ref. [2]. The present article reviews positron experimental techniques and some recent results from the positron facilities at Lawrence Livermore National Laboratory (LLNL).

\section{Experimental M ethods}

Positron annihilation studies of bulk materials at LLNL consist primarily of two techniques: Positron Annihilation Lifetime Spectroscopy (PALS) and Orbital Electron Momentum Spectroscopy (OEMS). After a positron enters a

A pproved for public release; further dissemination unlimited 
material, it quickly thermalizes in 110 psec. The positron will then diffuse in the material, and may become trapped in a localized state. Trapping or localization of positrons may occur in defects or other features in a material. Eventually, the positron annihilates with an electron, yielding two $511 \mathrm{keV}$ gamma rays.

In PALS, the positron lifetime in a material is determined by timing the interval between implantation of the positron into a material and its annihilation. Longer positron lifetimes indicate larger openvolume regions in a material. The intensity of each lifetime signal also gives an indication of the concentration of different openvolume regions. This method has been used to determine the heat of formation of vacancies in a material and localization of positrons at shallow traps [1], if the experimental apparatus is equipped with a method to control temperature of the specimens.

In OEMS, the energies of the two gamma rays produced after the annihilation of a positron with an electron are measured. Due to the momentum component of the electron positron pair in the direction of the gamma ray emission, these gamma rays are Doppler shifted, one blue shifted and one red shifted by an amount $\Delta E=p_{L} c / 2$, where $\mathrm{p}_{\mathrm{L}}$ is the longitudinal component of the electron positron momentum in the direction of the gamma ray emission and $c$ is the speed of light. This can be detected with two germanium detectors in coincidence[3]. Each element has a characteristic orbital electron momentum spectrum, resulting from the combination of core and valence electron momentum. Core electrons are more tightly bound to atoms and have a higher electron momentum than valence electrons, making the high momentum region of the OEMS useful for elemental specificity. By theoretically calculating the orbital electron momentum spectra or measuring the orbital electron momentum spectra of pure elements, the elements in the vicinity of the annihilating positron in multicomponent materials can be determined.

Two experimental facilities exist at LLNL for PALS and OEMS. A high-energy positron beam is formed using positron emitted from a ${ }^{22} \mathrm{Na}$ source placed at the terminal of a $3 \mathrm{MeV}$ electrostatic accelerator [1]. The positron beam (with a typical intensity of $5 \times 10^{5}$ positrons/s) emerging from the accelerator column is focused by a thin solenoid and exits the vacuum through a $25 \mu \mathrm{m}$ thick Al window. The positrons enter a specimen either directly or after passing through a $2 \mathrm{~mm}$ thick scintillator, which provides a start signal for positron lifetime measurements. The positron penetration depth is $\sim 1$ $5 \mathrm{~mm}$, and allows us to measure bulk properties without significant contributions from near-surface regions. The annihilation spectrum is detected with detectors positioned co-linear (for PALS) or 
perpendicular (for PALS and OEMS) to the positron beam direction. Since the beam exits the vacuum system, the setup is useful for characterizing a variety of samples including engineering materials with specialized fixtures, encapsulated samples requiring a specific environment, and liquids.

positrons have a mean kinetic energy of $\sim 200 \mathrm{keV}$, giving a penetration depth in materials of 30-100 $\mu \mathrm{m}$. This setup allows OEMS studies of gases and the magnetic properties of materials; magnetic measurements are performed by using the natural spin polarization of positrons emitted from a radioactive source.

\section{Results}

\section{Bulk Metallic Glasses}

Over the past 10 years, a new class of metallic glass forming alloys has been developed. These bulk glass forming alloys require cooling rates as low as $0.1 \mathrm{~K} / \mathrm{s}$ [6-8] to form a glass, whereas previous metallic glass forming alloys required cooling rates on the order of $10^{6} \mathrm{~K} / \mathrm{s}$ for glass formation. The ability to form a glass at lower cooling rates allows bulk components to be formed (a cooling rate of $10^{6} \mathrm{~K} / \mathrm{s}$ can only be achieved in very thin samples). These bulk metallic glass-forming alloys have useful engineering properties (high elastic limit, high yield strength) as well as providing unique processing opportunities for metallic components[9].

The second experimental facilisy is a setup similar to the one originall

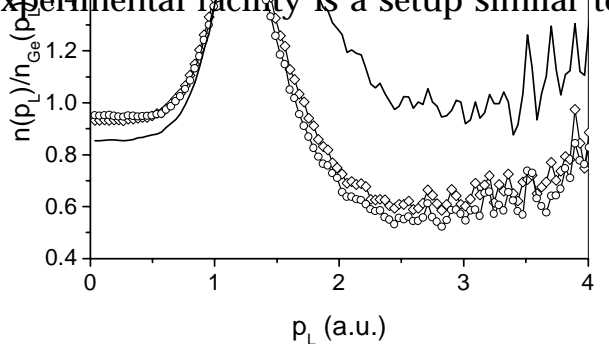

Figure 1. OEMS of the $\mathrm{Zr}_{52.5} \mathrm{Ti}_{5} \mathrm{Al}_{10} \mathrm{Cu}_{17.9} \mathrm{Ni}_{14.6}$ bulk metallic glass forming alloy in both the amorphous and the crystal line states. The line (atomic \%) corresponds to a linear combination of individual elemental spectra weighted by the atomic composition of the alloy.

PAS has been used in metallic glass studies of free volume changes with temperature[10,11], the chemical order around free volume regions[12], and the effects of hydrogen on viscoelastic relaxation[13]. A review of all of this work can be found in Ref. [14].

As-quenched bulk metallic glass samples exhibit a single lifetime, which is shorter than the lifetime of vacancies in an elemental metal yet longer then the crystalline form of the metals from which the specific metallic glass is made. There is a small change in positron lifetime with crystallization of the metallic glasses, which is evidence of 
positron localization in specific sites in the metallic glass (trapping).

The OEMS of amorphous (as prepared by casting) and crystallized (produced with a $650^{\circ} \mathrm{C}, 1 \mathrm{~h}$ anneal) $\mathrm{Zr}_{52.5} \mathrm{Ti}_{5} \mathrm{Al}_{10} \mathrm{Cu}_{17.9} \mathrm{Ni}_{14.6}$ [15] is shown in Fig. 1. For the x-axis, momentum, pL, is given in atomic units (a.u., 1 a.u. = $1.85 \mathrm{keV}$, with $\left.\mathrm{p}_{\mathrm{L}}=2 \Delta \mathrm{E} / \mathrm{c}\right)$. The OEMS in this figure are all divided by the OEMS of germanium; i.e., $n\left(p_{L}\right) / n_{G e}\left(p_{L}\right)$. This is done in order to emphasize differences in the spectra. The experimental OEMS for the amorphous and crystallized samples do not agree with the atomic \% OEMS. The atomic \% OEMS is a calculated curve that corresponds to the linear combination of elemental OEMS in which the positron wave function is sampling a perfectly random distribution of the elemental constituents.

Fits to the experimental data were constructed by calculating linear combinations of elemental OEMS, adjusting the coefficients to obtain a good fit. Figure 2 shows a comparison of these coefficients, giving a measure of the atomic constituents surrounding the open volume area in which the positron annihilates. The $\mathrm{Ni}$ and $\mathrm{Cu}$

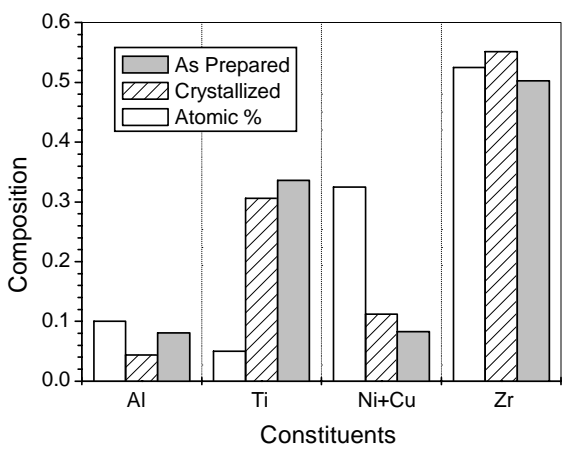

Figure 2. Elemental composition surrounding open volume areas of the $\mathrm{Zr}_{52.5} \mathrm{Ti}_{5} \mathrm{Al}_{10} \mathrm{Cu}_{17.9} \mathrm{Ni}_{14.6}$ bulk metallic glass forming alloy.

contributions to the OEMS are combined due to their elemental OEMS being very similar to each other, making it difficult to resolve their separate contributions. The two largest differences from the atomic \% OEMS are a larger $\mathrm{Ti}$ contribution and a smaller $\mathrm{Ni}+\mathrm{Cu}$ contribution. The $\mathrm{Ti}, \mathrm{Zr}$, and $\mathrm{Al}$ surround open volume regions, while $\mathrm{Ni}$ and $\mathrm{Cu}$ reside in close packed regions. Short-range order is present in this bulk metallic glassforming alloy. Crystallization of the alloy does not significantly change the atomic distribution surrounding open volume regions.

PALS measurements on this alloy yielded a lifetime of $177 \pm 3$ ps, while the known ordered phases in a crystallized sample have calculated lifetimes of $143 \mathrm{ps}\left(\mathrm{NiZr}_{2}\right)$ and $151 \mathrm{ps}$ $\left(\mathrm{Zr}_{2} \mathrm{Cu}\right) . \quad \mathrm{Al}_{3} \mathrm{Zr}_{4}$ has a calculated lifetime of 177 ps, but the annihilation of positrons in this crystalline phase can be discounted 
due to the large $\mathrm{Ti}$ signal in the OEMS.

The low momentum fraction ( $p_{\mathrm{L}}<0.38$ a.u.) of the OEMS versus temperature for the $\mathrm{Zr}_{52.5} \mathrm{Ti}_{5} \mathrm{Al}_{10} \mathrm{Cu}_{17.9} \mathrm{Ni}_{14.6}$ alloy is shown in Figure 3. The low momentum fraction is a measure of the number of positron annihilating with delocalized, low-momentum electrons, present in open volume regions of a sample; the larger the open volume region, the greater the low momentum fraction. Larger open volume regions, due to lack of positively charged ionic cores, will also have a larger binding energy for positions. As this specimen is cooled, the low momentum fraction initially increases and then decreases at $\mathrm{T} \sim 225 \mathrm{~K}$. This is evidence for the existence of at least two different sized open volume regions in this bulk metallic glass-forming alloy.

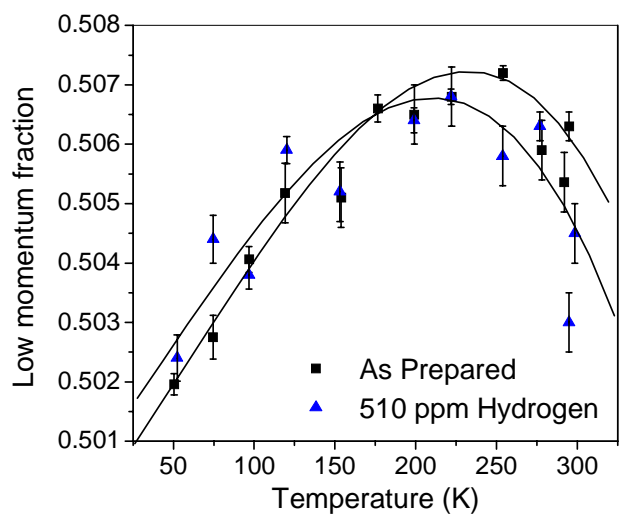

Figure 3. Temperature response of the low momentum annihilation fraction showing the detrapping of positions at room temperature and the contribution of shallow traps at low temperatures.
At room temperature, positrons are localizing (trapping) in a larger open volume region. However, this open volume region does not strongly bind the positron at room temperature, so some detrapping occurs. As the temperature is decreased, the positrons in the specimen are more completely trapped in these open volume regions, as shown by an increase in the low momentum fraction. At $\mathrm{T} \sim 225 \mathrm{~K}$, the positrons do not have enough energy to detrap from this open volume region. The low momentum fraction decreases due to a high volume fraction of smaller open volume regions in the sample beginning to trap the positrons when the temperature is reduced below $\mathrm{T} \sim 225 \mathrm{~K}$.

Also shown in Figure 3 is data from the same $\mathrm{Zr}_{52.5} \mathrm{Ti}_{5} \mathrm{Al}_{10} \mathrm{Cu}_{17.9} \mathrm{Ni}_{14.6}$ alloy that has been charged with hydrogen. The hydrogen localizes to larger open volume regions in the sample, as shown by the shift of the peak in the low momentum fraction to a lower temperature of $\mathrm{T} \sim 200 \mathrm{~K}$. This shift is due to the hydrogen filling the open volume region, reducing the positron binding energy and the volume fraction of this open volume region. Also, there is a reduced population of large open volume holes as shown by the reduction in low momentum fraction at room temperature.

\section{Nuclear Reactor}

\section{Pressure Vessel Steels}


The continued operation or lifetime extension of a number of nuclear power plants around the world requires an understanding of the damage imparted to the pressure vessel steel by irradiation. This damage results in a high number density of nanometer-sized copper rich precipitates and sub-nanometer defect-solute clusters, which are the cause of irradiation embrittlement.

Nanometer sized coppermanganese-nickel rich precipitates have been identified as the primary embrittling feature in reactor pressure vessel (RPV) steels with greater than 0.05 to $0.1 \% \mathrm{Cu}$ [16]. However, questions still remain regarding the composition of these precipitates, with small angle neutron scattering (SANS) and atom probe tomography (APT) yielding different results. A secondary embrittling feature has been identified as three-dimensional, subnanometer vacancy-solute copper complexes, called stable matrix features. The vacancy character of these secondary features has been inferred from simulation results and annealing experiments.

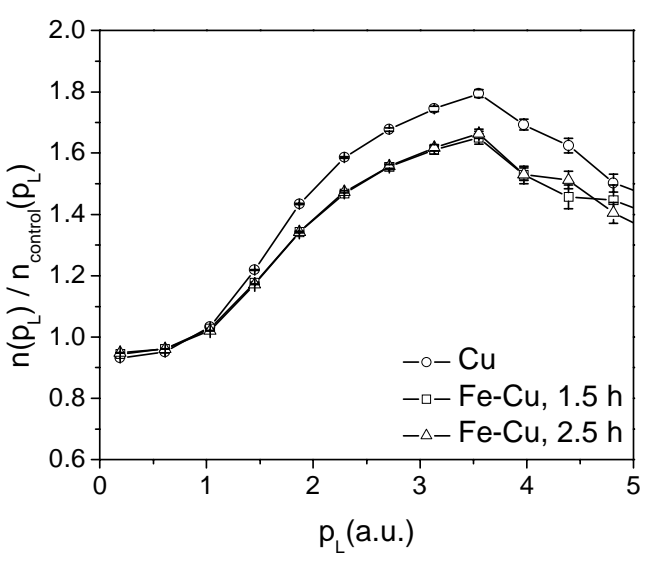

Figure 4. Normalized OEMS for $\mathrm{Fe}$ $1.5 \mathrm{wt} \% \mathrm{Cu}$ thermally aged at $500^{\circ} \mathrm{C}$ for $1.5 \mathrm{~h}$ and $2.5 \mathrm{~h}$. Positron annihilation in Fe would correspond to a value of 1 (i.e., a horizontal line at 1 ) in this plot.

In the RPV steels, consisting of iron, carbon, and other impurities, positrons will preferentially localize in defect regions, which have a larger open volume. After the steels have been heat treated or irradiated, nanometer sized copper-manganesenickel precipitates form (vacancies and vacancy clusters are also present in the irradiated material, in addition to the copper-manganese-nickel precipitates). Copper has a higher positron affinity than iron $(\mathrm{Cu},-4.81$ $\mathrm{eV}, \mathrm{vs}$. Fe, -3.84 eV [2]), so positrons will preferentially localize in the copper precipitates. This confinement of positions by nanoscale sized copper precipitates in a thermally aged $\mathrm{Fe}-1.0 \mathrm{wt} \% \mathrm{Cu}$ alloy was first shown by Nagai et. al. [17].

The OEMS of two thermally aged Fe-1.5 wt \% $\mathrm{Cu}$ alloys are shown in Figure 4 [18], in addition to 
a reference spectrum of pure, wellannealed elemental copper. These samples were annealed and quenched to keep the copper in supersaturated solution, followed by thermal aging at $500^{\circ} \mathrm{C}$ to allow copper to form precipitates. The spectra in this figure are divided by a spectrum from the control specimen; the control specimen is the same alloy that was annealed and quenched but not thermally aged. The positrons are confined almost exclusively to the copper precipitates, as shown by the OEMS qualitatively matching the OEMS of copper.

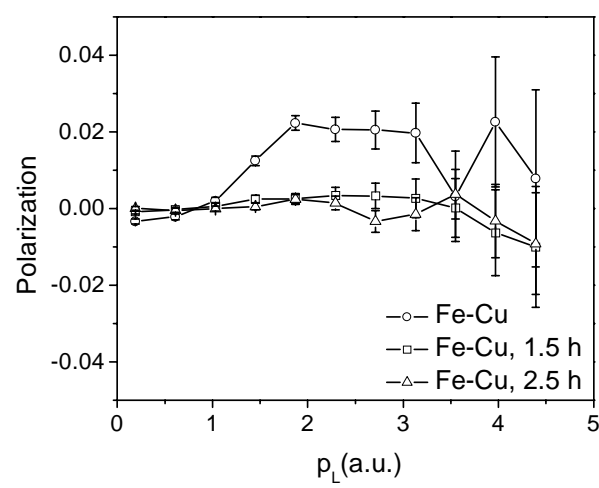

Figure 5. Electron polarization spectrum as a function of orbital electron momentum for Fe-1.5 wt\% $\mathrm{Cu}$ annealed and thermally aged at $500^{\circ} \mathrm{C}$ for $1.5 \mathrm{~h}$ and $2.5 \mathrm{~h}$.

The magnetic character of the copper precipitates in the iron matrix can also be measured, giving an indication of the amount of iron within these copper precipitates [19]. Positrons annihilate with electrons of opposite spin, yielding two gamma rays; if the spins are parallel, three gamma rays are emitted, which will not be recorded with the two detectors in coincidence. In the ACAR like positron setup at LLNL, both the positron source and the specimen are in a magnetic field that is used to guide the positrons to the specimen. The positron spin is parallel to its momentum; by changing the polarity of the magnetic field, the spins of the electrons in the specimen will change direction. The polarization is defined using the equation $P\left(p_{L}\right)=\frac{n_{\uparrow}-n_{\downarrow}}{n_{\uparrow}+n_{\downarrow}}, \quad$ where $n_{\uparrow}$ and $n_{\downarrow}$ are the number of electrons (after normalization) that annihilate (producing two gamma rays) in the specimen when the magnetic field is antiparallel or parallel, respectively, to the spin of the positrons. An example of these measurements is shown in Figure 5. The annealed Fe$1.5 \mathrm{wt} \% \mathrm{Cu}$ specimen, in which copper precipitates have not formed, exhibits a broad peak, starting at 1 a.u. momentum. The heat-treated specimens, however, exhibit relatively little difference with a change in the magnetic field polarization, showing that the precipitates are not magnetic, and therefore have minimal iron content.

Figure 6 shows the OEMS of a series of $\mathrm{Fe}-0.9 \mathrm{wt} \% \mathrm{Cu}$ alloys that have been irradiated under different fluxes/ fluences at $290^{\circ} \mathrm{C}$ (Table 1) [20], in addition to a reference spectrum of pure, well-annealed elemental copper. Even at the lowest irradiation condition (i.e., A2), copper precipitates are present, showing that there is rapid 
nucleation of copper precipitates. With increasing fluence, the low momentum portion of the spectrum ( $p_{\llcorner}<1$ a.u.) is increasing, with the broad peak at $p_{\mathrm{L}} \cong 3.5$ a.u. decreasing in intensity. These changes in the spectra are consistent with vacancy clusters forming in the alloy (increase in $\mathrm{p}_{\mathrm{L}}<1$ a.u.), and $\mathrm{a}$ competition between the relative efficiency of positron trapping in copper precipitates versus positron tapping in vacancy clusters, along with vacancy cluster density.

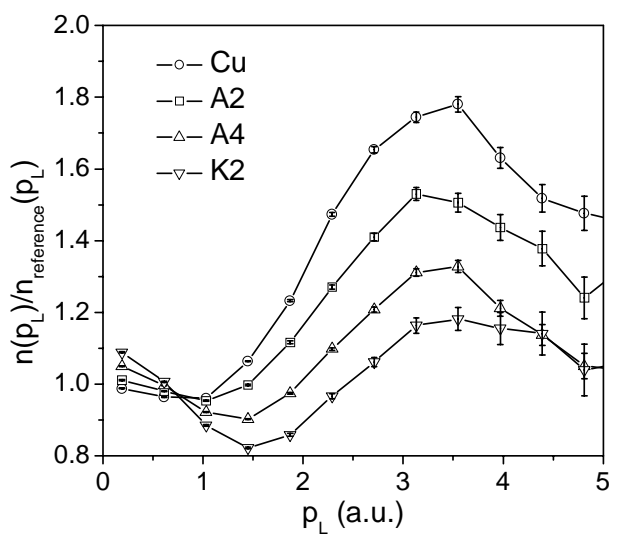

Figure 6. Normalized OEMS for a series of $\mathrm{Fe}-0.9 \mathrm{wt} \% \mathrm{Cu}$ alloys that have been irradiated under different fluxes/ fluences at $290^{\circ} \mathrm{C}$ (Table 1).

The trapping of positrons in vacancy clusters is confirmed by the PALS. The control specimen has a single lifetime component of 108 ps while the irradiated samples have two or three lifetime components, indicative of defect populations. The A2 specimen, irradiated at a fluence of $1.0 \times 10^{21} \mathrm{n} / \mathrm{m}^{2}-\mathrm{s}$, exhibits two lifetime components, corresponding to the growth of a vacancy cluster (lifetime component 2). The A4 specimen exhibits three lifetime components, with the 226 ps component growing in intensity compared to the $A 2$ specimen, and an introduction of a third lifetime component, corresponding to a larger vacancy cluster. The A5 specimen, with a fluence very close to that of the A4 specimen, has similar lifetime components to that of the A 4 specimen.

\section{Zeolites}

Zeolites are a class of hydrated aluminosilicate minerals that contain alkali and alkaline-earth metals. They have a framework structure with a series of interconnected cavities; larger metal cations and water molecules occupy these cavities. Zeolties, with a high surface area to volume ratio, are commercially used for the separation of hydrocarbons in petroleum refining, the drying of gases and liquids, and pollution control by selective molecular adsorption.

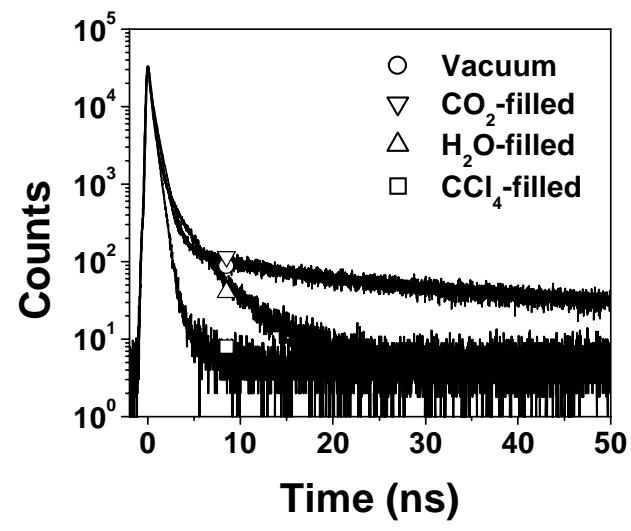

Figure 7. Plot showing the lifetime spectrum from Zeolite-Y samples when filled with different molecules. 
We have completed a series of experiments on a set of Zeolite-Y samples after baking in vacuum to remove water and other gaseous contaminants. Zeolite-Y has a faujasite type framework structure, with three different cavities or cages. It is widely used in the petroleum industry as a cracking catalyst.

The resulting positron lifetime data show a clear separation into two distinct sample sets based on the cation form and the Si/ Al ratio. We obtained lifetime components centered at 0.6-0.8 ns and 3-4.5 ns, with an additional longer lifetime component (30-45 ns) associated with positronium in the large intergranular regions. Surprisingly, there is no lifetime component at the value expected ( 2 and 8 ns) for the cage structures in Zeolite-Y. We suggest that the observed lifetime value of 3-4.5 ns is the average of these two cage structures. In order to test these lifetime assignments, we measured the changes in lifetimes when three molecules of different sizes (and hence different cage occupancies) are introduced into the samples. As shown in figure 7, when the Zeolite-Y is filled with $\mathrm{CCl}_{4}$, a molecule that can enter the larger cage structure but not the smaller one, a lifetime component consistent with positronium sampling of the smaller cage structure is observed at $1.8 \mathrm{~ns}$.
Positron annihilation lifetimes and electron momenta determined by the coincidence Doppler broadening technique were both determined for a set of alkali halide samples known to emit positronium into vacuum. Orbital electron momentum measurements examined the chemical specificity of the positron annihilation sites in alkali halides. The quality of our samples is determined by the consistency of our measured positron lifetime values with previous results on high quality samples. The electron momentum data at high momentum values indicate the ionic species that are contributing electrons for the annihilation. Figure 8 shows the electron momentum spectra obtained from several insulating materials with a halogen as one of the constituents. The momentum spectra are normalized to a reference element, $\mathrm{Ge}$, to highlight the atom-specific momentum-dependent variations. The top panel shows results from crystals with fluorine as a common element, while the bottom panel shows potassium compounds in which halogen is varied from fluorine to iodine. Momentum distributions from crystals containing fluorine show a broad peak centered at 1.3 atomic units, while results from crystals containing $\mathrm{Cl}, \mathrm{Br}$, and I show different behavior with less pronounced peak and centroids shifted to lower momentum values.

\section{Alkali Halides}




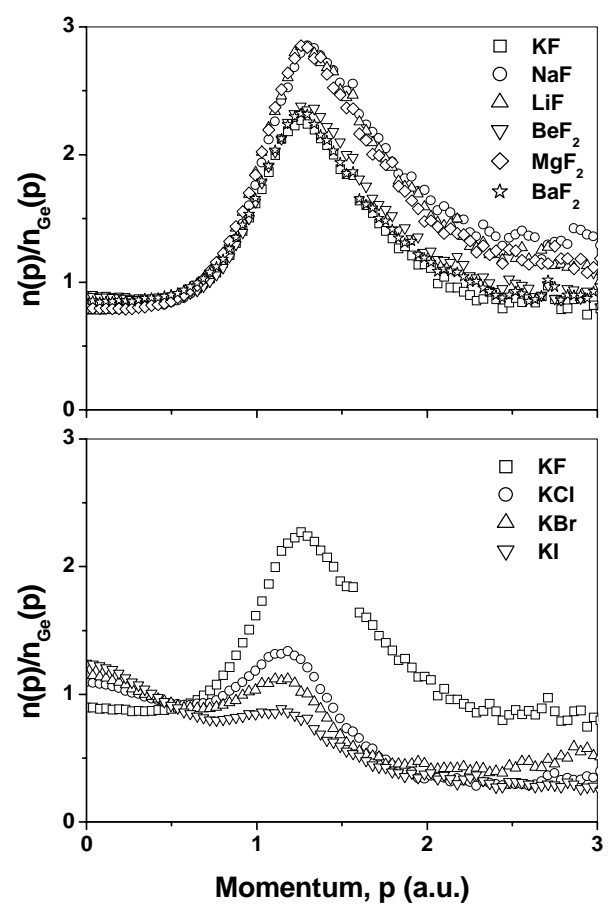

Figure 8. Electron momentum distributions for several alkali halide and alkaline earth-halide compounds plotted as a ratio to $\mathrm{Ge}$. These results are consistent with positron annihilation with only the halide electrons.

Our experimental results indicate that the annihilation is essentially entirely associated with the anion electrons. Existing theories that include the possibility of both cation and anion annihilation do not explain these results. The experimental observation of essentially $100 \%$ overlap of the positron with the halide atoms becomes even more significant when we recall that positronium, a neutral state, dominates the positron state in these materials. For this selectivity to occur in a positronium state either the positronium system is highly polarizable, leading to selftrapping, or the positronium is trapped at a structural defect surrounded by halide atoms, e.g. an alkali vacancy.

\section{Summary}

We provided several examples of recent positron studies of bulk materials using positron lifetime and electron momentum measurements.

\section{Acknowledgements}

This work was preformed under the auspices of the U.S. Department of Energy by the University of California, Lawrence Livermore National Laboratory under contract No. W-7450ENG-48, with partial support provided by Basic Energy Research, Division of Materials Science. We would like to acknowledge useful discussions with $\mathrm{R}$. Howell and A. Bug.

\section{References}

1. Positron in Solids, Topics in Current Physics, Vol. 12, Springer, Heidel berg, P. Hautojärvi (Ed.) 1979.

2. M.J. Puska and R.M. Nieminen, Rev. Mod. Phys. 66, 841 (1994).

3. P. Asoka-Kumar, M. Alatalo, V.J. Ghosh, A.C. Kruseman, B. Nielsen, and K.G. Lynn, Phys. Rev. Lett., 77, 2097 (1996).

4. R.H. Howell, P.A. Sterne, J. Hartley, T.E. Cowan, J. App. Surf. Sci., 149, 103 (1999).

5. "Positron Studies of the Electronic Structure of Solids", R.N. West, in Proceedings of the International School of Physics, Course CXXV, Positron Spectroscopy of Solids, eds. A. Dupasquier and A.P. Mills jr. (IOS Press, A msterdam, 1995), pp 75.

6. A. Inoue, T. Zhang, and T. Masumoto, Mater. Trans. JIM 31, 425 (1990).

7. A. Peker and W.L. Johnson, Appl. Phys. Lett., 63, 2342 (1993).

8. J. Schroers and W.L. Johnson, Appl. Phys. Lett., 80, 2069 (2002).

9. For more information, see the web site, http:/ / www.liquidmetal.com/

10. C. Nagel, K. Rätzke, E. Schmidtke, J. Wolff, U. Geyer, and F. Fraupel, Phys. Rev. B 57, 10224 (1998). 
11. C. Nagel, K. Rätzke, E. Schmidtke, F. Faupel, and W. Ulfert, Phys. Rev. B 60, 9212 (1999).

12. P. Asoka-Kumar, J. Hartley, R. Howell, P.A. Sterne, and T.G. Nieh, A ppl. Phys. Lett., 77, 1973 (2000).

13. D. Suh, P. Asoka-Kumar, and R.H. Dauskardt, A cta Mater. 50, 537 (2002).

14. K.M. Flores, D. Suh, R.H. Dauskardt, P. A soka-Kumar, P.A. Sterne, and R.H. Howell, J. Mater. Res. 17, 1153 (2002).

15. X.H. Lin, Ph.D. Thesis, California Institute of Technology (1997).

16. G.R. Odette and G.R. Lucas, J. of Metals 53, 18 (2001).

17. Y. Nagai, M. Hasegawa, Z. Tang, A. Hempel, K. Yubuta, T. Shimamura, Y. Kawazoe, A. Kawai, and F. Kano, Phys. Rev. B 61, 6574 (2000).

18. S.C. Glade, B.D. Wirth, P. A sokaKumar, P.A. Sterne, S. Jumel, and P. Pareige, "Characterization of nanostructural features in thermally aged and irradiated Fe-Cu-Mn-Ni reactor pressure vessel model alloys," To be published (2002).
19. P. Asoka-Kumar, B.D. Wirth, P.A. Sterne, R.H. Howell, and G.R. Odette, Phil. Mag. Lett. in print (2002).

20. S.C. Glade, B.D. Wirth, P. AsokaKumar, P. A. Sterne, , R.H. Howell, and G.R. Odette "Effect of Manganese on the of Evolution of Radiation Damage in Reactor Pressure Model Alloys," To be published (2002).

Table 1. Irradiation conditions $\left(290^{\circ} \mathrm{C}\right)$ for a series of Fe 0.9 wt $\% \mathrm{Cu}$ alloys.

\begin{tabular}{|c|c|c|}
\hline & flux $\left[\phi\left(n / m^{2}-s\right)\right]$ & fluence $\left[\phi t\left(n / m^{2}-s\right)\right]$ \\
\hline$A 2$ & $7.0 \times 10^{14}$ & $1.0 \times 10^{21}$ \\
\hline A4 & $7.0 \times 10^{14}$ & $3.2 \times 10^{21}$ \\
\hline A5 & $5.0 \times 10^{15}$ & $4.0 \times 10^{21}$ \\
\hline $\mathrm{K} 2$ & $7.7 \times 10^{15}$ & $5.1 \times 10^{22}$ \\
\hline
\end{tabular}


Table 2. Positron annihilation lifetimes for the series of $\mathrm{Fe}-0.9 \mathrm{wt} \% \mathrm{Cu}$ alloys that have been irradiated under different fluxes/ fluences at $290^{\circ} \mathrm{C}$ (Table 1 ).

\begin{tabular}{|l|c|c|c|c|}
\hline Specimen & $\begin{array}{l}\text { Mean } \\
\text { lifetime } \\
<\tau>\text { (ps) }\end{array}$ & $\begin{array}{l}\text { Lifetime } \\
\text { component } \\
\mathbf{1} \\
\tau(\mathbf{p s}) / / \\
(\%)\end{array}$ & $\begin{array}{l}\text { Lifetime } \\
\text { Component } \\
\mathbf{2} \\
\tau \text { (ps)/I (\%) }\end{array}$ & $\begin{array}{l}\text { Lifetime } \\
\text { Component } \\
\mathbf{3} \\
\tau(\mathbf{p s}) / / \text { (\%) }\end{array}$ \\
\hline Control & 108 & $108 \pm / 100$ & & \\
\hline A2 & 137 & $112+2 / 78$ & $222+6 / 22$ & \\
\hline A4 & 165 & $111+7 / 61$ & $226 \pm 33 / 35$ & $401+93 / 5$ \\
\hline A5 & 153 & $110 \pm 6 / 65$ & $201+32 / 28$ & $351+35 / 8$ \\
\hline
\end{tabular}

